\title{
An Advanced Middleware Architecture for Secure and Survivable Mobile C4I Systems
}

\author{
Mr. Randy Browne \\ New Jersey Computer \& Communications \\ 2001 U.S. Highway 46, Suite 310 \\ Parsippany, New Jersey 07054
}

\author{
Mr. Jon Valénte \\ USAF Research Laboratory \\ Information Directorate / IFGA \\ 525 Brooks Road \\ Rome, New York 14850
}

\author{
Dr. Salim Hariri \\ University of Arizona \\ Center for Advanced TeleSysMatics \\ 1230 East Speedway Boulevard \\ Tucson, Arizona 85721
}

\begin{abstract}
This paper gives an overview of a middleware-based mobile C4I architecture that provides a foundation for balanced treatment of the complex, and frequently conflicting dependability requirements (i.e. security, survivability, etc.) of tactical systems. The architecture is an outgrowth of work on a mobile distributed operating system that attempts to deal with various shortcomings of the Mobile Internet Protocol (Mobile IP) for military use. The architecture provides secure and survivable mobile $\mathrm{C4I}$ operation by using a highly controversial technique - via Session Layer "hand off" of mobile communications - a function that is traditionally controlled entirely by the Network Layer. The architecture is described in functional terms, as well as the general layered structure that provides tactical dependability. Also described is an emerging idea known as Plan-Based Survivability, which the authors hope will solve many open problems with current survivability technology, and which has already been partly demonstrated by a working prototype.
\end{abstract}

\section{INTRODUCTION}

"Economic pressures obviously continue to force the Defense Community to increase reliance on commercial-offthe-shelf (COTS) information and communications technology. The private sector and the Defense Community share a common interest in fault tolerance, high availability, etc.. However, the private sector has no economic incentive to solve the most egregious security and survivability problems faced by future military mobile C4I systems. U.S. military adversaries, equipped with resources that far exceed typical private sector capabilities for industrial espionage, either have, or could obtain the potential to launch sophisticated attacks against mobile U.S. C4I assets that simultaneously exploit both physical and "cyberspace" C4I vulnerabilities.

There seems to be a widely held, tacit belief that different dependability mechanisms (e.g. defenses for physical assets versus information assets) can operate largely independently in different system layers. We strongly dispute this point of view, especially where mobile C4I operations are involved. While we agree that a layered C4I architecture is both possible and necessary, the layers of dependability

-This research was sponsored in part by the U.S. Air Force Research Laboratory under Contract F30602-96-C-0140. The opinions expressed are those of the authors and do not necessarily reflect official positions or policies of the United States Government.

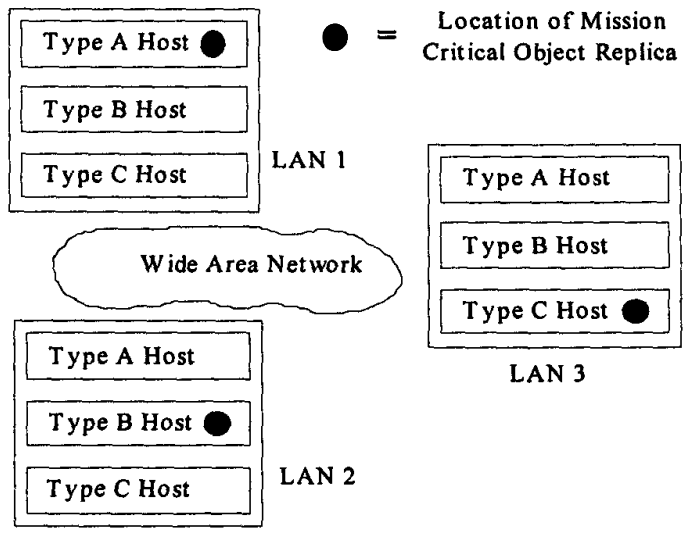

Fig. 1. Optimal Survivability of a 3-Quorum

mechanisms and defenses must interact to a much greater extent than most technologists seem to believe. The Defense Community needs a coherent overall architecture that can fuse the disparate paradigms of dependability mechanisms (e.g. using AI techniques versus formal methods to address different dependability problems). This paper discusses these issues and describes an emerging architecture that should substantially improve the state-of-the-art for dependable mobile C4I.

\section{COMPLEX DEPENDABILITY REQUIREMENTS}

It is well known that dependability requirements and mechanisms (e.g. expensive data replication protocols, security controls, etc.) are in conflict with system performance. Contemporary research [6] is just beginning to solve the dependability problems of complex systems having interconnected sub-systems with conflicting dependability requirements.

Interrelated dependability issues require coordinated defense mechanisms to thwart attacks. Fig. 1 shows a simple interdependence between information survivability and physical survivability. For the sake of illustration, we assume in Fig. 1 that the LANs are sufficiently geographically separated so that any individual physical attack destroys at most one LAN, given a hypothetical adversary's capabilities. Also, Host Types A, B, and C are assumed to have distinct 
information warfare (IW) vulnerabilities, that are equally difficult for an adversary to exploit. Under these assumptions, Fig. 1 shows an arrangement of three critical data object replicas that optimizes survivability (i.e. maximizes the number of available replicas) under simultaneous physical and IW attack.

Fig. 1 provides a hint as to why IW defenses must be coordinated with defensive systems for physical C4I assets. A defensive IW system might (properly) choose to place three critical object replicas (Fig. 1) on different host types, to provide technical diversity and complexity against IW attack. However, if the IW defenses operate independently from C4I physical defenses, then unlike what is shown in Fig. 1, the IW defensive system could mistakenly place all replicas on the same LAN, whereby all replicas could be lost in a single physical attack. Such an approach to C4I defense (using totally independent IW and physical defense systems) could be exploited by a clever adversary to defeat the overall $\mathrm{C} 4 \mathrm{I}$ system. By combining sequential IW and physical assault, an attacker might use computer viruses and worms to trigger software reconfiguration mechanisms which, in turn, leads to the accumulation of certain critical data items in a comparatively small number of physical sites. The adversary might then eliminate a quorum of the remaining data replicas via clandestine sabotage or overt physical attack. The simple point being made by Fig. 1 is that in order to optimize survivability of a maximum number of data object replicas, coordinated attacks can only be defeated by coordinated defenses. There is more to protecting information systems than just information warfare. C4I survivability entails simultaneous optimization with respect to both physical and "cyberspace" threats.

There can also be interdependencies between security and survivability. What is not shown in Fig. 1 is that one might use random migration to help hide the physical location and/or logical configuration of information assets to thwart an attack. In such a C4I system, even if there is no classified information per se, the system cannot be survivable if network traffic analysis [11] leads to discovery of asset locations. Focusing on survivability but ignoring security is pointless in such cases.

In sum, distinct $\mathrm{C} 4 \mathrm{I}$ dependability properties such as physical survivability, information survivability, integrity, service assurance, and security are not "orthogonal", contrary to widely held views. There is even an example in [2] that shows for certain real-time communications systems, it is mathematically impossible to determine whether some behavior anomalies are integrity violations, or denials of service, given only a "black box" system specification. For many dependability issues, different $\mathrm{C} 4 \mathrm{I}$ defenses must carefully cooperate to avoid vulnerabilities at the interface between the different defenses. Mobile C4I operation is an added complication to the dependability problem - at the very least, because of the added technical difficulties in maintaining position secrecy for mobile units involved

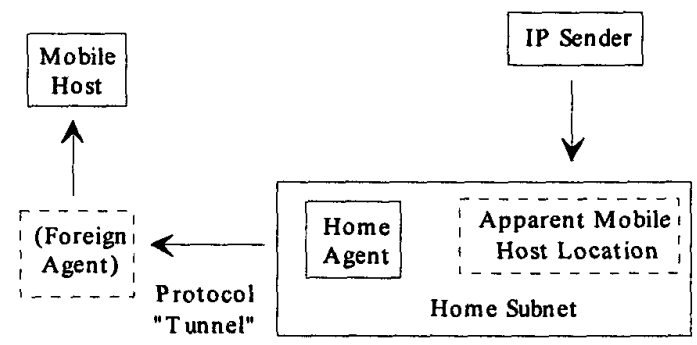

Fig. 2. The Mobile Internet Protocol

specifically in data communications, and the attendant impact on overall C4I survivability.

\section{THE "TETHERING" PROBLEM}

The C4I architecture we present later is motivated partly by the authors' attempts to fix some well-known problems in using the Mobile Internet Protocol (Mobile IP) in tactical settings. The problems we discuss with Mobile IP are not specific to Internet technology, but apply to a large class of mobile communication protocols that employ tunneling through legacy infrastructure. Our discussion is also relevant to emerging Ad Hoc network protocols [7], but we defer discussion of Ad Hoc networking until later.

Details of Mobile IP and proposed modifications and variants are given in $[8,10]$, and current specifications can be found in on-line Internet documentation. Fig. 2 sketches the basic operation of Mobile IP for our purposes, showing how an IP packet is routed from an arbitrary sender to a mobile receiver. The IP sender (and legacy routers not shown) would see the mobile host location as being fixed on the so-called home subnet (Fig. 2) associated with the mobile host's IP address. Mobile IP employs a home agent at the home subnet which transparently tunnels packets out a foreign agent, and then to the mobile host's current location [8].

The mobile host is thus "tethered" to its home subnet. Such "tethering" is generally no problem for the private sector, since it is feasible to implement a "hot spare" for the home agent which can take over if the home agent fails passively - adequate "survivability" for most commercial business uses. For military mobile C4I purposes however, the entire home subnet could be a LAN, which can be readily lost in combat, simultaneously destroying all primary and backup home agents. The possibility of being "tethered" to lost subnets can make a mobile host permanently unreachable no matter where it moves. Schemes do exist for bypassing the home subnet temporarily, by caching the location of the mobile host in IP routers [8]. However, such schemes only help efficiency and do nothing for survivability since, when router caches become stale, the home subnet/agent(s) must be consulted to update IP addresses in routers. Thus, the home subnet must always be reachable for survivability purposes.

One might consider some variants of Mobile IP or restrictions on its use to fix such problems. Physical 
survivability would mainly be a problem with LANs on ground vehicles, ships, and aircraft, that typically exist in the Internet address space as Class $\mathrm{C}$ subnets. A direct approach to fixing survivability problems caused by "tethering" mobile hosts to subnets would be to do something to the underlying subnet itself to make it a survivable entity, such as by distributing the subnet nodes (home agents, etc.) over wide geographic areas. However, requiring geographic dispersion of components in a large number of military LANs is generally impractical for a variety of obvious technical and administrative reasons.

Alternatively, one might devise a Mobile IP variant that could dynamically select from one of several home subnets, so that a mobile host can be dynamically "tethered" to any one of the available subnets. The IP address of a mobile host is thus a virtual IP address that can be dynamically bound to a number of "physical" IP addresses [10]. A suggestion by Sunshine and Postel [9] is to reserve certain IP addresses for mobile hosts. Use of reserved IP addresses in datagrams could trigger address translation (location finding) using a replicated directory service, for example. Such suggestions definitely improve survivability at least somewhat.

However, we have a fundamental complaint with all such proposals. When mobile survivability is at issue, the IP/Network Layer, and even the Transport Layer are simply the wrong layers of protocol to make the choice of home subnet. In a complex mobile C4I environment, as objects migrate between moving hosts, and as tactical mission priorities and objectives change in combat, survivability needs alone could justify changing the home subnet. The Network and Transport Layers do not handle enough of the right kind of information to make intelligent decisions about the best home subnet.

In object-oriented C4I systems, the Session Layer is the lowest layer of protocol that can be conversant about mission priorities, risks, asset valuations, etc.. Thus, the Session Layer is in a much better (even if not perfect) position to make the choice of home subnet for survivability purposes. However, we also want to dispense with the idea of a home subnet, to obtain an approach that is not tied to the Internet, and can deal with a variety of legacy Network and Transport protocols. This motivates our middleware mobile C4I architecture in which the Session Layer performs mobile communications "hand off", whether or not the Network Layer also does so.

\section{A DEPENDABle MobILE C4I ARCHITECTURE}

We give a brief overview of the major components of a mobile C4I architecture (Fig. 3) that addresses the key problems discussed earlier. We cannot give more than just a very cursory system description in a paper of this scope the reader is referred to $[3,4]$ for details. We only give sufficient information to make a general comparison of our work with related developments in mobile communications.

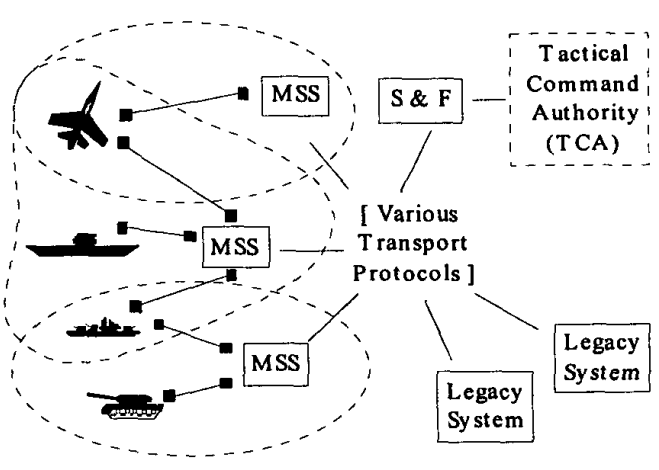

Fig. 3. Basic Mobile C4I Sy stem Components

Fundamentally, our system is an object-oriented, clientserver system. For the time being, the labeled, boxed nodes shown in Fig. 3 are assumed to be stationary - that is, from the Session Layer perspective, the Network topology and Transport quality of service for the "boxed nodes" (only) in Fig. 3 do not change as a result of any motion they might have. We note that a geostationary satellite could be "stationary" in this sense. The concept of Plan-Based Survivability discussed later will permit us to relax the restriction that some network nodes must be stationary. For now, we assume the existence of a stationary supporting infrastructure as a starting point for discussion, since many mobile computing environments (e.g. the mobile Internet) are extensions of legacy stationary infrastructure.

The dashed areas in the left of Fig. 3 are called Zones of Coverage, or just zones, and are regions for mobile systems to access our C4I infrastructure. Thus, our architecture is superficially similar to a cellular network, but the reader is cautioned against drawing too direct of a parallel with a conventional cellular network. Like cells, zones can overlap or not, and can be distinguished, in principle, by geographic region, or by time and/or frequency division communication, etc. in any combination. Unlike cells however, zones are Session Layer abstractions; not of the Network Layer. Zones are primarily Transport address domains, so a Transport address is only valid in a given zone. Only object identifiers (i.e. Session addresses in the object-oriented sense) have endto-end significance. Also, zones may, but do not necessarily correspond 1-1 to a "broadcast radius" for some transmitter. Zones can be a regional internetwork of mixed wireless and wired infrastructure extended by "broadcast repeaters" Session Layer zones could even consist of multiple cells of a Network Layer cellular network.

Each zone is controlled by a Mobile Session Server (MSS). Each mobile host is supported by a Store-and-Forward (S\&F) facility that coordinates motion across zones. The role of the Tactical Command Authority (TCA) in Fig. 3 will be described later. 


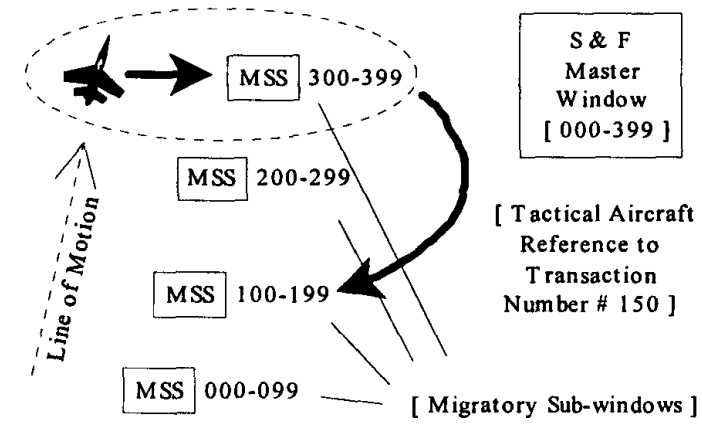

Fig. 4. Nominal Motion-Induced Session Behavior

\section{TRANSPORT LAYER BEHAVIOR}

System behavior at the Transport Layer is fairly straightforward. When a mobile host (e.g. aircraft, tank, ship, etc. in Fig. 3) moves into a zone, the MSS controlling that zone assigns a Transport address to the mobile host that is only meaningful within the zone. In fact, TCP/UDP over Mobile IP is a reasonable choice for the Transport Protocol used within a single MSS zone - but not across zones, because of the aforementioned "tethering" problem. A mobile host can maintain different Transport addresses for different zones. For example, an aircraft (upper left of Fig. 3) may have two Transport addresses at the same time assigned by two separate MSS devices controlling a pair of overlapping zones covering the aircraft's current position.

While in a given zone, since most communication is interzone, an MSS sets up one or more dynamic Transport bridges for use by a mobile host, based on object references (in the object-oriented sense) made by the mobile host. After the mobile host leaves an MSS zone, the Transport bridges are deactivated.

\section{SESSION LAYER BEHAVIOR}

System behavior at the Session Layer is more complex. Each mobile host performs Session Layer communication using a conventional "sliding window" protocol - with the caveat that the Session Peer of the mobile host is the entire C4I system, and not any specific entity [4]. Referring to Fig. 4, each mobile host maintains a Master (Protocol) Window that is managed by the Store-and-Forward (S\&F) facility and defines the currently allowed range of Session Message sequence numbers for individual outstanding objectoriented method invocations/transactions. To support survivability, the Master Window is partitioned into migratory sub-windows that are managed by MSS hosts, and which can be independently migrated and recovered (Fig. 4). As with other mobile object-oriented middleware systems [14], proxy objects are dynamically activated on MSS hosts to manage sub-windows when needed.
To create a new object method invocation/transaction, the issuing mobile host assigns a Session Message sequence number to the transaction from within the current Master Window. The Master Window on the S\&F facility (Fig. 4) is used as a directory to locate the MSS that manages the subwindow containing the given sequence number. To reduce the frequency of access to the S\&F-resident Master Window, the MSS hosts use a cache of sub-window locations [4]. When the mobile host is not directly in the zone of a managing MSS (e.g. for request \# 150 in Fig. 4), other MSS hosts will form dynamic Transport bridges to extend the request/reply path from the mobile host to the managing MSS. If it is necessary to activate a sub-window not currently managed by an MSS, the S\&F facility chooses a site. The site selection protocol is described in [4], but generally, MSS sites are selected by (1) using motion-history data to choose candidate MSS hosts recently known to be near the mobile host, and by (2) obtaining command approval from the TCA (Fig. 3) for use of a particular site.

After a mobile host leaves an MSS zone, the Session Layer (sub-window proxy) objects do not promptly terminate unlike the Transport Layer functions. They generally persist for some time, usually a matter of minutes, and continue to perform mobile host proxy services as done in systems such as [14]. Unlike systems such as [14], our proxy objects migrate periodically using heuristics that try to balance load and keep those objects near the mobile host - all subject to command approval by the TCA. Sub-windows and their (complex) migration behaviors are transparent to mobile hosts. Fig. 4 shows a benign case where a mobile host generates a series of method invocations, in generally sequential order, and thus leaves an "activation trail" of subwindow proxy objects as it moves from zone to zone.

\section{RATIONALE FOR SESSION LAYER MOBILE "HAND OFF"}

The architecture described in Fig. 3 and Fig. 4 avoids the "tethering" problem discussed earlier. There isn't any notion of a permanent "home subnet" at the Network or Transport Layer. Moreover, below the Session Layer, no protocol address has any end-to-end significance. When an MSS is lost for any reason, only the Transport addresses and bridge services for that zone are lost. At the Session Layer, MSS loss triggers recovery procedures to restore/migrate Session sub-window proxy objects to other active MSS nodes [4]. Survivability of the S\&F facility is achieved via replication and (using Plan-Based Survivability technology discussed later) by migration.

Functionally, as mobile hosts move across MSS zones, our architecture changes the Transport addresses of mobileresident objects. In effect, we are performing mobile "hand off" at the Session Layer. During informal discussions with colleagues, we've found this to be the most controversial aspect of our architecture, so some comments are in order. The primary point is that for the sake of survivability, the C4I 
Session Layer is required to routinely participate in mobile "hand off".

To see why, consider the information available at different protocol layers. An intelligent mobile Network or Transport Layer could extract certain survivability-related information from their protocol addresses. For example, a Network address may uniquely identify the underlying host platform, operating system, etc. and perhaps even the physical location of the host. From this information, the Network Layer might derive information about threats to the host - e.g. "cyberspace" vulnerabilities of a particular operating system or hardware type, and risk of conventional attack due to physical location, etc.. However, what the Network and Transport Layers cannot know (without regular intervention by higher protocol Layers), is asset value information. That is, the Network and Transport Layers cannot know (1) the current or future value to friendly forces of information on a network host, nor (2) the future value of the hardware assets themselves based on needs to support future missions.

By contrast, the Session Layer of an object-oriented C4I system has potential access to a rich set of information about the tactical arena. Object-oriented $\mathrm{C} 4 \mathrm{I}$ applications can place vast amounts of tactical information (e.g. about threats, asset values, tactical plans, etc.) in object containers - as a socalled tactical framework. The Session Layer, which among other things actually implements the object abstraction over the network, can access information within the tactical framework in the same form available to the C4I Application Layer - via method invocations on C4I objects. The possibility of sharing a common tactical framework between the Session Layer and Application Layer means that the Session Layer can perform its traditional functions in a way that addresses survivability, yet still is generally transparent to the Application Layer. For example, one traditional Session Layer function is to form client-server "bindings" for replicated objects - choosing an object server from among those available to a given client. The Session Layer can select an object server from a sub-quorum most likely to survive in support of some critical C2 function, often just by deriving or obtaining such information from the shared tactical framework - without otherwise interacting with the Application Layer. The tactical framework could similarly provide survivability-related information for other Session Layer functions, such as choosing destination hosts for object migration, controlling sizes of replication quorums, managing process group memberships, etc.

The connection between the tactical framework and mobile communications is twofold. First, in order to make sound survivability decisions, the Session Layer functions need some geometry/topology information to have a complete tactical picture. Some of the needed information is available down in the mobile Network Layer, but might be obtained from other sources. Second, survivability-sensitive Session Layer functions need information about service dependencies in lower protocols layers, in order to determine whether critical C2 functions are indirectly dependent on any directory services, "home networks", or other lower layer mechanisms within vulnerable parts of the infrastructure.

The basic design problem for mobile C4I systems, is that under traditional protocol structure, neither the Session, Transport, nor Network Layers has all of the information needed to support survivability decisions. Perhaps we need to completely rethink our traditional notions of protocol layering to properly accommodate survivable mobile computing, but that is a complex matter we cannot address in this paper. In any case, for an object-oriented C4I system, the Session Layer is far closer to having complete information than the lower layers given the aforementioned possibility of a tactical framework (shared with the Application Layer) that can provide much of the missing information. The fundamental question is how to implement the traditional Session Layer functions in light of the way information is distributed between layers? In brief, for reasons of simplicity and portability, we decided to "elevate" to the Session Layer some of the high-level geometry information normally hidden by the mobile Network Layer. Our division of the C4I infrastructure into MSS zones (Fig. 3) provides the Session Layer with coarse-grained information about geometry and topology for tactical information and physical assets.

The inter-zone communications, established by a series of dynamic Transport bridges, represent high level routes for which the Session Layer can address survivability requirements in an integrated manner (Fig. 1). MSS zones generally represent enclaves that differ in their survivability characteristics. Our Session Layer thus bears an analogous relationship to the Transport and mobile Network Layers that the Internet bears to its subnets. It is not our intent to replace the mobile "hand off" functions of the Network Layer. Rather, our architecture with its MSS zone structure provides the Session Layer with a high level, virtual and survivable network for which it could make coarse-grained survivability decisions - using a C4I tactical framework that would be meaningless to the Transport and Network Layers. However, the fact that our architecture can obviate the need for Network "hand off" in some cases (e.g. when MSS zones are implemented by large overlapping regions) has led to some controversy. Nonetheless, Session Layer "hand off" is a reasonable way to provide geometry/topology information in support of Session Layer survivability mechanisms.

\section{AD HOC NETWORKS}

Mobile communications protocols (e.g. Mobile IP) generally attempt to provide some kind of "addressstabilization" (address invariance under motion) using "tunneling" [8], directory services [9], and/or other mechanisms. A general characterization of the "tethering" problem might be that for the sake of survivability, mobile hosts must not be "tethered" to any "address-stabilization" mechanism that is implemented by a vulnerable part of the infrastructure, unless the mechanism can be readily recovered such as by migration, reconfiguration, etc.. 


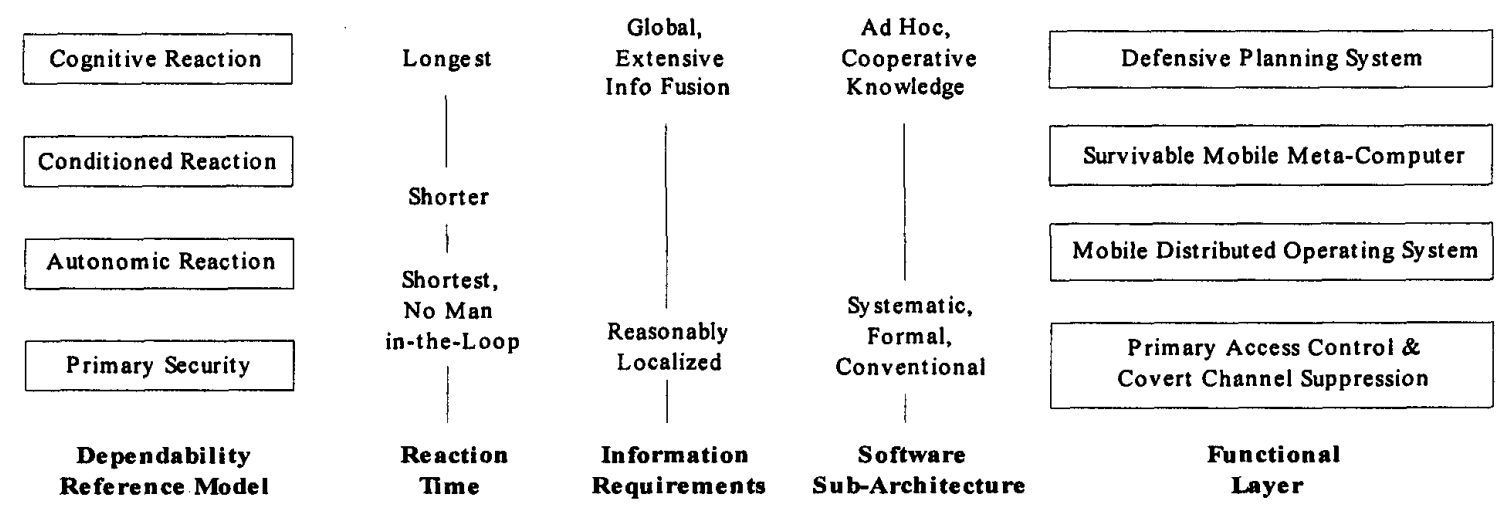

Fig. 5. Plan-Based Survivability: Model and Expected Functional Structure

In the informal sense just defined, there is no survivabilityrelated "tethering" problem with "pure" Ad Hoc network protocols [7]. In an Ad Hoc network, there are typically no specialized hosts with special functions relating to mobility, and a case could be made that "best effort" peer connectivity is adequate support for survivability at the Session Layer. We would generally agree, and the Session Layer "hand off" performed by our architecture is probably redundant to Ad Hoc network functions in such cases, except that, depending on the scope of the Ad Hoc network, the Session Layer might need to impose certain route restrictions.

However, survivability problems similar to those we described for Mobile IP could arise in Ad Hoc networks, when extending Ad Hoc networks to scalable infrastructure. For example, one might need a dynamic directory service (a specialized host function) to find a mobile host within a complex internetwork of multiple Ad Hoc enclaves. In such cases, "tethering" problems associated with the directory service or whatever mechanism could arise - for survivability, the use of "home bases", "well known addresses", etc. should be avoided where the mechanisms are vulnerable, and aren't migratory/reconfigurable. Thus, for interconnected Ad Hoc networks, our earlier comments would apply - the Session Layer must exert control over the configuration of inter-enclave communications, because as discussed earlier, the (inter-Ad-Hoc) Network Layer protocol would not be in a position to determine proper defensive action in the tactical environment. Designers of future Ad Hoc network protocols should keep in mind the need for Session Layer control of enclave extension mechanisms, in order to optimize overall mobile C4I system survivability.

\section{FUTURE DIRECTIONS: PLAN-BASED SURVIVABILITY}

So far, we have partly described what can be regarded as a mobile distributed operating system - an object-oriented "platform" for physically mobile hosts, and logically mobile objects. We have not yet explained how the Tactical Command Authority (TCA) in Fig. 3 performs its function which is to approve, disapprove, and even to change the recommendations for object placement/migration made by the S\&F facility. Moreover, the TCA approves/disapproves all object placement/migration choices made by C4I applications and other parts of the system not shown in Fig. 3.

The criteria that the TCA uses to approve or disapprove placement of a C4I object on a host is based on an emerging technical paradigm we call Plan-Based_Survivability. The concept of Plan-Based Survivability is still an emerging research area, so we can only outline general technical directions in this paper.

Overall, Plan-Based Survivability means the use of tactical planning information to guide the real-time search for a mapping from information assets to physical assets in a way that is simultaneously optimal with respect to all threats (e.g. physical, IW, administrative, personnel security, etc.).

Fig. 5 illustrates Plan-Based Survivability. The far left of Fig. 5 shows a layered Dependability Reference Model (DRM) which is implemented by the functional layers shown at the right of Fig. 5. The Layers of the DRM are intended mainly as points on a continuum and do not correspond exactly to the more well-defined functional layers at the right of Fig. 5.

The lowest DRM layer is the Primary Security Layer. As the name implies, while there are security mechanisms in all layers of the model, the main host and network security mechanisms are at the base of the system, in accordance with conventional views of multilevel distributed system security [11]. A key function of this layer is location-secrecy for C4I objects.

Above the primary security mechanisms are the sub-layers of the survivability part of the model. The lowest such layer is the Autonomic Reaction Layer which provides rapid, mostly automatic "damage control" using information fairly local to the point of attack. The next layer is the Conditioned Reaction Layer, which has somewhat longer response times, but protects information and physical assets according to preexisting, well-defined tactical plans. The highest layer of the DRM is the Cognitive Reaction Layer, which has the longest reaction time, but provides the most sophisticated response. 
Cognitive reactions are effected by global fusion of threat and damage assessment data and are carried out by distributed knowledge sources, agent-based mechanisms, etc.. The DRM Layers avoid a "one-scheme-fits-all" problem in terms of both information warfare and conventional attack.

Referring to the far right of Fig. 5, Plan-Based Survivability is realized by functional layers that generally (but do not exactly) correspond to the DRM. The lowest functional layer implements multilevel security. Access control mechanisms, as well as new technologies for "covert channel suppression" [11] are used to ensure location secrecy of migrated objects (where necessary) over and above the security policy for the system. Technologies within this layer are based on Mode Security [1] and related technologies [13]. Phenomena that disclose C4I object locations can arise both from flaws in trusted operating systems $[1,11]$, and from traffic analysis and other communications behavior $[11,13]$.

The next functional layer (of a C4I system utilizing PlanBased Survivability - Fig. 5, far right) is a mobile distributed operating system, which is essentially the system we described in Fig. 3, excluding the TCA. (For comparison, the TCA in Fig. 3 generally corresponds to the top two functional layers at the upper right of Fig. 5.) The mobile distributed operating system implements essentially the autonomic, or "instinctive" reactions of the system, relying heavily on fully automated recovery mechanisms. The mobile distributed operating system provides an object-oriented medium for the operation of higher layers. The tactical framework mentioned earlier that is shared between the Application and Session Layers would consist of C4I objects implemented by the mobile distributed operating system.

The next layer is a survivable. mobile meta-computer (Fig. 5, far right, center). This layer fuses the various C4I computing and communications assets into a C4I "supercomputer" for solving the complex optimization problems that arise in searching for optimal survivability, as well as other $\mathrm{C} 2$ problems we don't address in this paper. The meta-computing layer will probably be an enhanced version of the Virtual Distributed Computing Environment [12]. The meta-computer generates and stores various Combined Asset Protection Plans (CAPPs - not shown in Fig. 5) which are used to approve or disapprove migration/activation of objects (Fig. 3) on various C4I hosts. CAPPs that are approved by higher command functions (discussed next) represent the "conditioned reactions" for defending information assets under the DRM. CAPPs are "combined plans" in that they deal with both physical and information assets to ensure optimal survivability against all threats, as illustrated by our discussion of Fig. 1.

The top functional layer is the Defensive Planning Layer and primarily implements the "cognitive reactions" of the DRM. This layer controls the meta-computing layer to produce and approve various CAPPs. There are various planning systems that could serve as the basis for the Defensive Planning Layer.
Further research should illuminate the pros and cons of Plan-Based Survivability, but we generally outline expected benefits here. Many key survivability issues are inherently domain-specific [2], and domain-specific knowledge is known to be useful for constraining the search over complex solution spaces for many optimization problems. In our case, the problem domain is distributing information assets (objects) to computing and communications devices to maximize survivability from all C4I threat sources including physical and "cyberspace" threats. Plan-Based Survivability provides domain constraints so that survivability mechanisms and functions are tied to mission success objectives. Plan-Based Survivability is also a "lessonlearned" from the history of AI research. To paraphrase and extend a convincing point from [5], significant gains in $\mathrm{AI}$ were made in the 1970 s by postponing the search for general AI technology, in favor of effective AI technology - even if only useful in limited domains, initially. More general AI technology (e.g. machine learning) evolved later in the 1980s and beyond, owing much to 1970 s AI technology (e.g. expert systems and knowledge representation) for specific problem domains. In order to stimulate development of relevant (even if not entirely general) survivability technology, Plan-Based Survivability is intended to provide the same kind of domainspecific focus to survivability, that expert systems historically provided for developments in AI.

Plan-Based Survivability is largely just an attempt to extend existing paradigms of defensive tactical planning to also cover the protection of information assets. In the opening of this paper, we alluded to the idea that current thinking tends to treat the $\mathrm{C} 4 \mathrm{I}$ information infrastructure as a separate, ubiquitous layer that somehow will independently maintain adequate availability under various tactical situations. With Plan-Based Survivability, the idea of combining information protection with other $\mathrm{C} 4 \mathrm{I}$ asset protection is actually a more "conservative" view of high technology - in effect, we regard information and associated technology as a mere extension of the commander's logistic support system. Under Plan-Based Survivability, the various C4I computers, communications, and information are formally protected only to the extent they support current and future mission objectives.

The benefits of adopting Plan-Based Survivability as a paradigm for mobile $\mathrm{C} 4 \mathrm{I}$ survivability are various. By using pre-planning as much as possible, efficiency is improved largely by reducing contention, much the way that prescheduling is used in hard real-time systems, and the way that pre-scheduling of voice communications channels is used for planning tactical aircraft missions. Computationally, C4I realtime algorithms at various layers would be designed to be "plan-centric", so the focus of real-time communications (especially the focus of data communications) becomes confirming hypotheses about how the actual "world-state" differs from the intended tactical "plan-state", which can be much more efficient than tactical communications that are not guided by some kind of planning model [4]. This is 
particularly important where only low throughput communications are available in certain forward areas, and where the electronic "footprint" of mobile systems must be kept as small as possible.

Plan-Based Survivability also provides a formal basis for taking preventive measures to protect information and other C4I assets. Tactical plans (CAPPs) should contain threatavoidance strategies triggered in response to real-time situation assessment information. Defensive IW techniques that only react after the onset of an attack could get overloaded by a rapid attack against large segments of the information infrastructure - the old adage that "an ounce of prevention is worth a pound of cure" applies to C4I information protection as much as anything.

Finally, Plan-Based Survivability can be used at various layers in a communications system to provide more "fluidity" and beneficial technical complexity against physical and IW attacks. CAPPs could include randomly generated preplanned object migrations, which could be tailored during mission execution, to increase an adversary's entropy about friendly C4I system state (e.g. migrating the S\&F facility and other services depicted in Fig. 3, along with supporting directory services, etc.).

\section{CONCLUSION}

We have presented a new architecture for dependable (i.e. survivable, secure) operation in a mobile C4I environment, with the paper discussion focused on unified information and physical survivability. The architecture is controversial in that the Session Layer performs the primary communications function of mobile "hand off", instead of relying exclusively on the Network Layer to perform this function. We defended our approach on the basis that where tactical survivability is paramount, mobile "hand off" must be carefully controlled by the Session Layer, even if not specifically performed by that layer. Popular private sector approaches (e.g. Mobile IP) attempt to provide a "virtually stationary" environment by use of address mappings which, except for performance impact, completely hide motion effects in the Network Layer. Such mobile networking approaches are unsuitable for military mobile $\mathrm{C} 4 \mathrm{I}$ use, unless they are modified/designed to carefully coordinate their resource-management facilities with the survivability mechanisms of the Session Layer.

The mobile $\mathrm{C} 4 \mathrm{I}$ architecture presented is part of an evolving paradigm for C4I survivability that we call PlanBased Survivability. In effect, Plan-Based Survivability is a "superstructure" we propose for unifying a diverse hierarchy of C4I defenses, both physical and informational. There are many existing or developing Defense systems that could implement or support one or more layers of this "superstructure".

A working prototype has been constructed [4] that demonstrates some of the basic concepts in this paper. The prototype corresponds to Fig. 3, except that (1) the prototype S\&F facility is non-replicated and non-migratory, (2) the transaction management/recovery capabilities of the MSS hosts are fairly limited at the moment, and (3) the tactical framework shared between the C4I Session and Application Layers is still being defined. The authors anticipate a series of progressively more complex demonstration prototypes. The ultimate aim is to provide a working platform for dependable mobile C4I over world-wide Defense infrastructure.

\section{REFERENCES}

[1] Browne, R., "Mode Security: An Infrastructure for Covert Channel Suppression", In Proceedings of the 1994 IEEE Symposium on Security and Privacy, May 1994.

[2] Browne, R., "Defining Survivability", In Proceedings of the $1^{\text {st }}$ IEEE Workshop on Information Survivability, San Diego, CA, February, 1997.

[3] Browne, R., "Mobile Computing in a Heterogeneous Distributed Computing Environment", Final Report for Contract F30602-95-C0188, USAF Rome Laboratory Technical Report RL-TR-96-252, March, 1997.

[4] Browne, R., "Mobile Computing in a Heterogeneous Distributed Computing Environment (Phase II)", Final Report for Contract F30602-96-C-0140, U.S. Air Force Research Laboratory, In Press.

[5] Forsythe, R., Ed., "Expert Systems: Principles and Case Studies", Q1984, Chapman and Hall Computing, ISBN 0-412-26270-3.

[6] Hinton, H., and Lee, E.S., "The Compatibitity of Policies", In Proceedings of the $2^{\text {nd }}$ ACM Conference on Computer and Communications Security, November, 1994.

[7] Haas, Z., "A New Routing Protocol for the Reconfigurable Wireless Networks", ICUPC'97, San Diego, CA.

[8] Johnson, D., "Scalable and Robust Internetwork Routing for Mobile Hosts", In Proceedings of the $14^{\text {th }}$ International Conference on Distributed Computer Systems, June, 1994.

[9] Sunshine, C., and Postel, J., "Addressing Mobile Hosts in the ARPA Internet Environment”, Internet Engineering Note IEN 135, March, 1980.

[10] Teraoka, F., Claffy, K., and Tokoro, M., "Design, Implementation, and Evaluation of the Virtual Internet Protocol", In Proceedings of the $12^{\text {th }}$ International Conference on Distributed Computer Systems, June, 1992.

[11] "Trusted Network Interpretation of the Trusted Computer System Evaluation Criteria", National Computer Security Center Document \# NCSC-TG-005, July 1987.

[12] Topcuoglu, H., Hariri, S., Kim, D., Kim, Y., Bing, X., Ye, B., and Valénte, J., "The Design and Evaluation of a Virtual Distributed Computing Environment", Technical Report, Syracuse University Dept of EE and CS, USAF Rome Laboratory Contract \# F30602-95-C-0104.

[13] Venkatraman, B., and Newman-Wolfe, R., "Capacity Estimation and Auditability of Network Covert Channels", In Proceedings of the 1995 IEEE Symposium on Security and Privacy, May 1995.

[14] Yeo, L. H., and Zaslavsky, A., "Submission of Transactions from Mobile Workstations in a Cooperative Multidatabase Processing Environment", In Proceedings of the $14^{\text {th }}$ International Conference on Distributed Computer Systems, June, 1994. 